\title{
Non-local Coulomb correlations in metals close to a charge order insulator transition
}

\author{
Jaime Merino \\ Departamento de Física Teórica de la Materia Condensada, \\ Universidad Autónoma de Madrid, Madrid 28049, Spain
}

(Dated: November 26, 2018)

\begin{abstract}
The charge ordering transition induced by the nearest-neighbor Coulomb repulsion, $V$, in the 1/4-filled extended Hubbard model is investigated using Cellular Dynamical Mean-Field Theory. We find a transition to a strongly renormalized charge ordered Fermi liquid at $V_{\mathrm{CO}}$ and a metal-toinsulator transition at $V_{\mathrm{MI}}>V_{\mathrm{CO}}$. Short range antiferromagnetism occurs concomitanly with the $\mathrm{CO}$ transition. Approaching the charge ordered insulator, $V \lesssim V_{\mathrm{MI}}$, the Fermi surface deforms and the scattering rate of electrons develops momentum dependence on the Fermi surface.

PACS numbers: 71.27.+a, 71.30.+h, 74.70.+Kn
\end{abstract}

Strongly correlated electronic systems in low dimensions exhibit novel quantum states of matter with unconventional electronic properties. Among the fascinating phenomena observed in these systems is the Mott metal-insulator transition induced by the local Coulomb repulsion energy [1] which occurs, for instance, in transition metal oxides and layered organic superconductors [2, 3]. Important progress in the description of the Mott transition [4] has been achieved with the development of dynamical mean-field theory (DMFT) [ [5], a local theory which predicts that close to the Mott insulator (MI), a quasi-particle peak and Hubbard bands coexist in agreement with photoemission spectra of transition metal oxides [6] and optical spectra of $\kappa$-(BEDT-TTF $)_{2} \mathrm{X}[7]$. Non-local correlations can modify this scenario opening a pseudogap in the spectral density as Cellular Dynamical Mean-Field Theory (CDMFT) [8] and the Dynamical Cluster Approximation (DCA) [9] have found consistent with the pseudogap phase of high- $\mathrm{T}_{c}$ superconductors.

The 1/4-filled family of layered organic molecular superconductors of the $\alpha, \beta^{\prime \prime}$ and $\theta$-(BEDT-TTF $)_{2}$ $\mathrm{X}$ types display rich phase diagrams 10 and anomalous metallic behavior [11] such as the Drude peak absence in the optical conductivity of $\beta^{\prime \prime}$-(BEDT-TTF $)_{2}$ $\mathrm{SF}_{5} \mathrm{CH}_{2} \mathrm{CF}_{2} \mathrm{SO}_{3}$ 12. In these quasi-two-dimensional systems both the on-site Coulomb repulsion, $U$, and the nearest-neighbor Coulomb repulsion $V$ are substantial. In spite of its importance, the electronic properties of metals close to a Coulomb-driven charge ordered insulator (COI) transition remain poorly understood. Taking the extended Hubbard model as the minimal strongly correlated model which includes the competition between itinerancy and localization due to charge ordering we study the properties of metals close to a COI. An important theoretical challenge is to understand the evolution of the spin degrees of freedom across the charge ordering transition and, in particular, whether spin and charge order together or not.

In this Letter, we describe how the COI transition driven by the Coulomb repulsion, $V$, in the extended
Hubbard model occurs based on the CDMFT approach, which is a strong coupling method which includes both on-site and short range non-local correlations. We find a transition to a strongly renormalized charge ordered metal $(\mathrm{COM})$ at $V_{\mathrm{CO}}$ and a metal-insulator transition at $V_{\mathrm{MI}}$. We find that the spectral density is suppressed on some regions of the Fermi surface due to the momentum dependence developed in the electron scattering rate close to the COI. Concomitanly with the $\mathrm{CO}$ transition an antiferromagnetic $(\mathrm{AF})$ interaction between spins at charge rich sites of the COM is dynamically generated by the Coulomb interaction, $V$.

The simplest strongly correlated model that captures the competition between charge ordering and the homogeneous metal is an extended Hubbard model on a square lattice at 1/4-filling:

$$
\begin{aligned}
H= & -t \sum_{\langle i j\rangle, \sigma}\left(c_{i \sigma}^{\dagger} c_{j \sigma}+c_{j \sigma}^{\dagger} c_{i \sigma}\right)+U \sum_{i} n_{i \uparrow} n_{i \downarrow} \\
& -\mu \sum_{i \sigma} c_{i \sigma}^{\dagger} c_{i \sigma}+\sum_{\langle i j\rangle} V_{i j} n_{i} n_{j},
\end{aligned}
$$

where $\sigma$ is the spin index and $c_{i \sigma}^{\dagger}$ and $c_{i \sigma}$ denote the electron operators. $t$ is the transfer integrals between nearest-neighbor sites of the square lattice. The Coulomb interaction is such that: $V_{i j}=V$ if $i$ and $j$ are nearest neighbor sites and $V_{i j}=0$ otherwise. The system with no interactions $(U=V=0)$ has a dispersion with bandwidth $W=8 t$. We fix $U=10 t$ and analyze the $\mathrm{CO}$ transition due to $V$ at $1 / 4$-filling.

In the CDMFT method, the infinite lattice is divided into identical clusters of size $N_{c}=4$ which are treated exactly as shown in Fig. 1 (a). The Coulomb interaction between electrons in different clusters is treated at the mean-field level. The quantum cluster is embedded in a non-interacting bath which is solved self-consistently. Results are presented for paramagnetic phases unless explictly stated. The effective Anderson model reads

$$
H=\sum_{\alpha k} \epsilon_{k}^{\alpha} n_{k}+\sum_{i \sigma} \epsilon_{i} n_{i \sigma}+\sum_{\alpha k i \sigma} \Gamma_{k i}^{\alpha}\left(c_{k \sigma}^{+} c_{i \sigma}+c . c .\right)
$$




$$
+U \sum_{i} n_{i \uparrow} n_{i \downarrow}+\sum_{i j} t_{i j}\left(c_{i \sigma}^{+} c_{j \sigma}+c_{j \sigma}^{+} c_{i \sigma}\right)+\sum_{i j} V_{i j} n_{i} n_{j}
$$

where $t_{i j}=-t$ denote hopping matrix elements between neighbor sites inside the cluster with $i$ and $j$ running from 1 to Nc. Following Ref. [13], bath levels are grouped into $\alpha$ subsets. Correspondingly the energies, $\epsilon_{k}^{\alpha}$, refer to the $k$ energy level of the $\alpha$ subset. The bath-cluster couplings between a bath level $k$ of bath $\alpha$ and the cluster site $i$ are: $\Gamma_{k i}^{\alpha}$. In practice, we truncate the bath to $N_{b}=8$ sites which are grouped into two subsets: $\alpha=1,2$ with $k=1, . ., 4$ states each. We take $N_{c}=4$ cluster sites. Lattice symmetries (allowing for checkerboard charge ordering) reduce the bath parameters to 4 independent bath levels: $\epsilon_{k}^{\alpha}=\epsilon_{k+2}^{\alpha}$, and hybridization bath parameters: $\Gamma_{k i}^{\alpha}=\delta_{k i} \Gamma_{i}^{\alpha}$, with $\Gamma_{i}^{\alpha}=\Gamma_{i+2}^{\alpha}$. Thus, we use 4 independent bath levels and 4 independent hybridization parameters to fully describe the bath. After extracting the selfenergy of the cluster, $\Sigma_{i j}\left(i \omega_{n}\right)$, for an arbitrary choice of $\epsilon_{i}$ 's and $\Gamma_{k i}$ 's the lattice Greens function is found by imposing the superlattice periodicity:

$G_{i j}\left(i \omega_{n}\right)=\sum_{\mathbf{K}}\left(\left(i \omega_{n}+\mu-\sum_{k} V_{i k}\left\langle n_{k}\right\rangle\right) \delta_{i j}-t_{i j}(\mathbf{K})-\Sigma_{i j}\left(i \omega_{n}\right)\right)^{-}$

where $t_{i j}(\mathbf{K})$ is the Fourier transform of the cluster hopping amplitudes and $\mathbf{K}$ the momentum wavevector in the reduced Brillouin zone of the superlattice. Matsubara frequencies, $\omega_{n}=(2 n+1) \pi / \beta$, with an inverse temperature: $\beta=200 / t$ are used. $\left\langle n_{k}\right\rangle$ is the average electronic occupation in nearest-neighbor sites in neighboring clusters. The one-dimensional version of model (11) has been previously studied with CDMFT[14]. The two-dimensional Hubbard model on a frustrated square lattice close to half-filling (with small degree of frustration), leads to an intricate competition between $\mathrm{AF}$, Mott insulator, unconventional superconductivity and metallicity [15].

The resulting phase diagram of the extended Hubbard model based on CDMFT is shown in Fig. 1. As $V$ is increased the system undergoes a sharp transition from an homogeneous metal (M) to a strongly renormalized charge ordered metal $(\mathrm{COM})$ at $V_{\mathrm{CO}} \approx 1.3 t$. The jump in the order parameter of the $\mathrm{CO}$ transition, $n_{12}=\left\langle n_{1}\right\rangle-\left\langle n_{2}\right\rangle$ is evident from Fig. 1 (b). Initially, for $V<V_{\mathrm{CO}}$, the system is weakly correlated as the quasiparticle weight $Z_{i}=\left(1-\partial \operatorname{Im} \Sigma_{i i}\left(i \omega_{n}\right) /\left.\partial \omega_{n}\right|_{\omega=0}\right)$ in charge rich $\left(Z_{1}\right)$ and charge poor $\left(Z_{2}\right)$ cluster sites is $Z_{1} \sim Z_{2}=0.7$ consistent with slave-boson theory [16]. The weakly correlated metal becomes a strongly renormalized Fermi liquid once charge ordering sets in with $Z_{1}=0.1$ at $V=1.5 t$ indicating localization of electrons at every other site of the lattice due to CO. At $V_{\mathrm{MI}} \approx 2 t$ a gap opens in the spectral DOS indicating a MI transtion. Previous direct Lanczos calculations on 20-site clusters predict a MI transition at $V_{\mathrm{MI}} \approx 2.2 t$ and indicated a

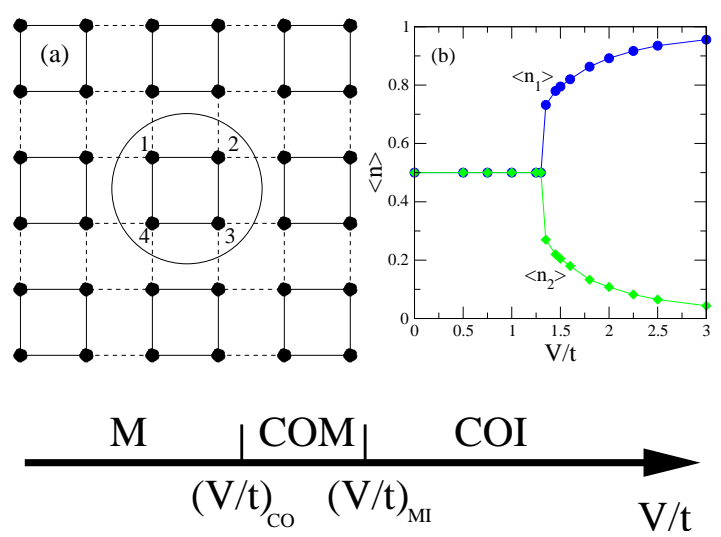

FIG. 1: (Color online) Ground state phase diagram of the 1/4-filled extended Hubbard model on the square lattice from CDMFT calculations. In (a) the tiling of the two-dimensional lattice used in CDMFT is shown whereas in (b) the $V$ dependence of site occupations, $n_{i}$, is plotted for $U=10 t$.

$\mathrm{COM}$ for $V>V_{\mathrm{CO}} \approx 1.5 t$ in remarkable agreement with - pur present results despite the small size of the cluster used within CDMFT.

Dynamical and short range correlation effects are analyzed based on cluster quantities: $G_{i j}\left(i \omega_{n}\right)$ and $\Sigma_{i j}\left(i \omega_{n}\right)$. Figs. 2 and 3 show how diagonal and off-diagonal selfenergies are enhanced for $V>V_{\mathrm{CO}}$ being most significant between charge rich sites (see $\Sigma_{13}\left(i \omega_{n}\right)$ in Fig. 3). Consistent with the self-energy enhancement, Green's functions are suppressed at finite $\omega_{n}$ for $V>V_{\mathrm{CO}}$. For $V \gtrsim 2 t, \operatorname{Im} G_{i i}\left(i \omega_{n} \rightarrow 0\right) \propto-\omega_{n}$, displaying insulating behavior [17]. This contrasts to the metallic-like behavior of $\operatorname{Im} G_{i i}\left(i \omega_{n}\right)$ for $V<V_{\mathrm{MI}}$ indicating a MI transition at $V_{\mathrm{MI}}=2 t$. Fig. 3 shows $\operatorname{Im} \Sigma_{i j}\left(i \omega_{n} \rightarrow 0\right) \rightarrow 0$ indicating that the opening of the gap is due to the large $\operatorname{Re} \Sigma_{13}\left(i 0^{+}\right)$ developing close to the COI (see Fig. 3). This interaction induced by Coulomb interaction produces a band splitting at the Fermi energy [18]. Note that as LRO-AF is not allowed, the opening of the gap at $V_{\mathrm{MI}}$ can only be due to short range correlations. We also find that at $V \approx 2.5 t, \operatorname{Im} \Sigma_{11}\left(i 0^{+}\right)$and $\operatorname{Im} \Sigma_{13}\left(i 0^{+}\right)$diverge.

The spectral DOS, $A_{i}(\omega)$, at site $i$ is shown in Fig. 4 indicating metallic behavior for $V<V_{\mathrm{MI}}$ while a gap is apparent at $V_{\mathrm{MI}} \approx 2 t$. The metallic phase can be further explored through the momentum resolved spectral DOS, $A(\mathbf{k}, \omega)$ with $\mathbf{k}$ being the momentum in the 1st Brillouin zone of the real lattice. Lattice self-energies are extracted by periodizing cluster selfenergies: $\Sigma(\mathbf{k}, \omega)=\frac{1}{N_{c}} \sum_{i} \Sigma_{i i}(\omega)+\Sigma_{12}(\omega) \cos \left(k_{x}\right)+$ $\Sigma_{14}(\omega) \cos \left(k_{y}\right)+\Sigma_{24}(\omega) \cos \left(k_{x}+k_{y}\right) / 2+\Sigma_{13}(\omega) \cos \left(k_{x}-\right.$ $\left.k_{y}\right) / 2$, with distances taken in units of the lattice parameter. The evolution of $A\left(\mathbf{k}, 0^{+}\right)$is displayed in Fig 5 which compares $V=t$ (a metal with no $\mathrm{CO}$ ) with $V=1.8 t$ (a COM close to the COI). For $V=t, A\left(\mathbf{k}, 0^{+}\right)$ describes a somewhat renormalized Fermi liquid and the 

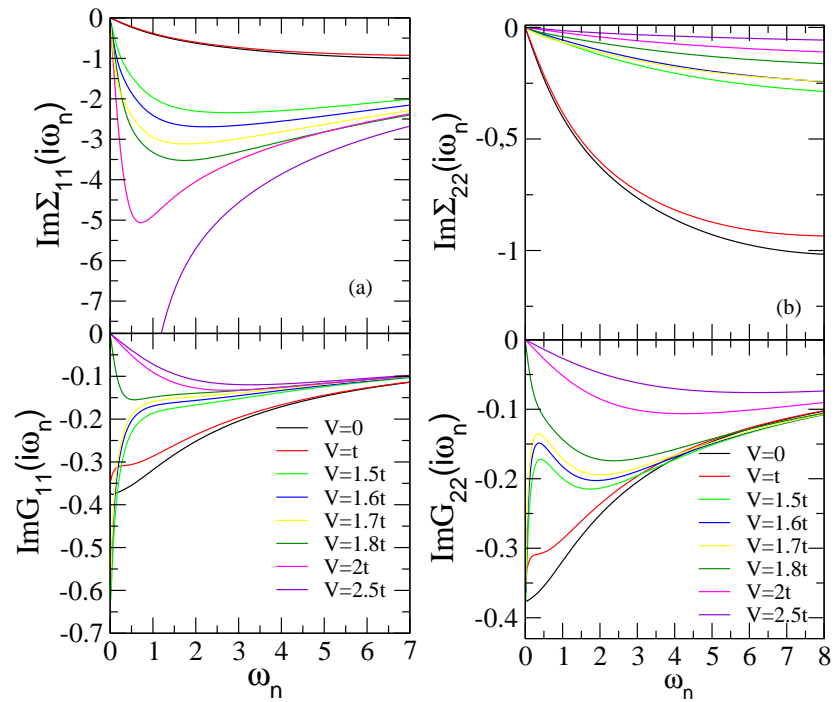

FIG. 2: (Color online) Enhancement of on-site self-energies across the CO and MI transitions with $V$. In (a) $\Sigma_{11}\left(i \omega_{n}\right)$ and $G_{11}\left(i \omega_{n}\right)$ which correspond to the charge-rich sites are plotted and in (b) the same for the charge-poor sites.
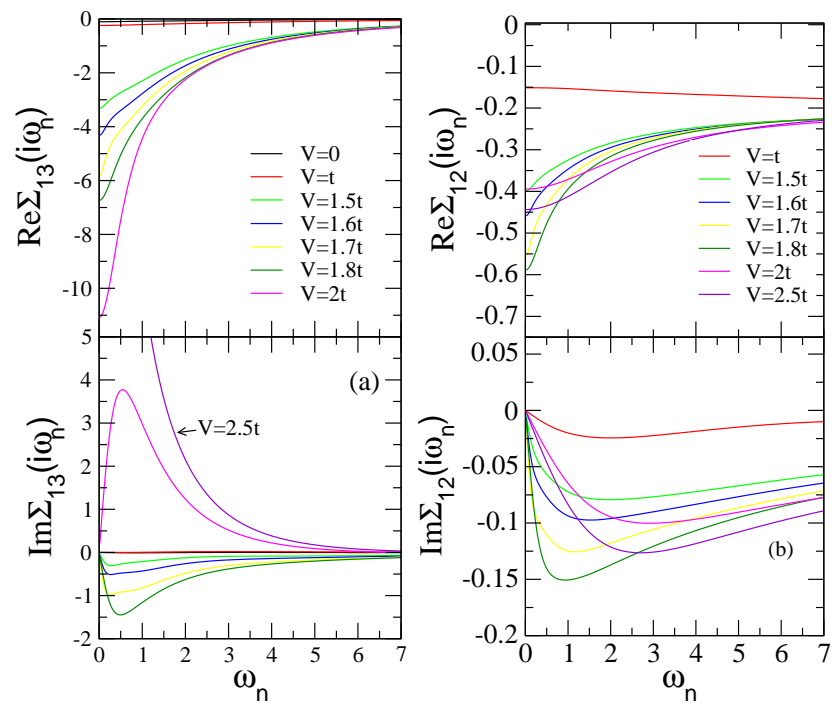

FIG. 3: (Color online) Dynamically generated off-site interaction in the COM. Non-diagonal self-energies are plotted with $V$. Self-energies $\Sigma_{i j}\left(i \omega_{n}\right)$ between two next-nearest neighbor charge rich sites are plotted in (a) while between nearestneighbor sites are displayed in (b).

non-interacting Fermi surface shape. For $V \lesssim V_{M I}$ the Fermi surface is deformed [19] and the scattering becomes momentum dependent. 'Cold' regions develop around momentum $\mathbf{k} \approx( \pm 0.4, \mp 0.4) \pi$ whereas 'hot' regions occur around $\mathbf{k} \approx( \pm 0.8, \pm 0.8) \pi$. This arises from the momentum dependence of the electron scattering rate: $1 / \tau_{\mathbf{k}}=-2 \operatorname{Im} \Sigma\left(\mathbf{k}, 0^{+}\right)$approaching the COI. In the top part of Fig. 5 the decrease of $1 / \tau_{\mathbf{k}}$ along the

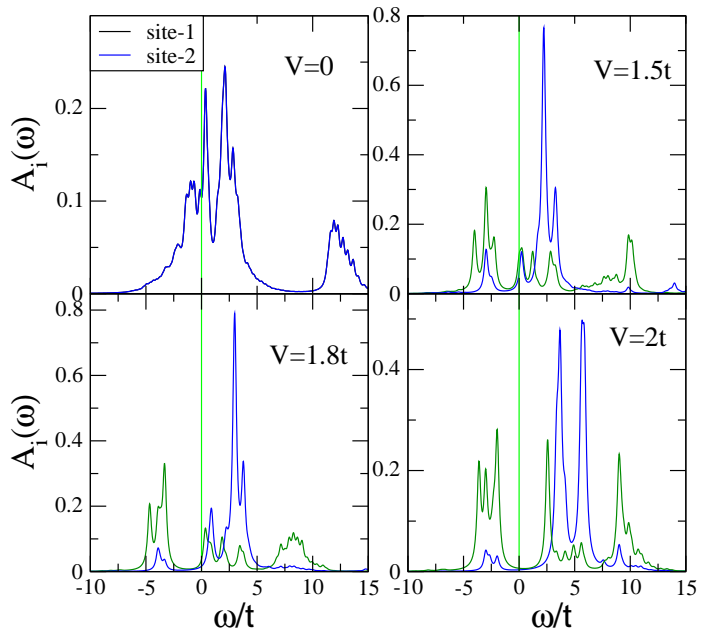

FIG. 4: (Color online) Evolution of the DOS with the Coulomb repulsion, $V$, across the CO transition. $A_{i}(\omega)$ is plotted for different $V$ showing a gap opening at the COI transition $V_{\mathrm{MI}}=2 t$. The vertical line denotes the position of the chemical potential.

$(0,0) \rightarrow(\pi,-\pi)$ direction is sharper for larger $V$ while along $(0,0) \rightarrow(\pi, \pi), 1 / \tau_{\mathbf{k}}$ remains large. The momentum dependence of $1 / \tau_{\mathbf{k}}$ leads to the anisotropic momentum dependence of $A\left(\mathbf{k}, 0^{+}\right)$. Although the precise position of 'cold' and hot 'regions' can vary for larger $N_{c}$ (as the momentum dependence of the self-energy is restricted by the symmetries of the small $N_{c}=4$ cluster), the momentum dependence of $1 / \tau_{\mathbf{k}}$ and Fermi surface deformation are robust features derived from CDMFT close to the COI. This is in contrast to single-site DMFT 20] which predicts a direct transition from a metal to a strongly correlated Fermi liquid with isotropic scattering rate for any $V$.

Magnetic properties are analyzed by computing the static spin correlation function, $\left\langle S_{i z} S_{j z}\right\rangle$, between different sites in the cluster. The spin interaction between charge-rich sites, $\left\langle S_{1 z} S_{3 z}\right\rangle$, is $\mathrm{AF}$ for all $V$ as shown in Fig. 6. At the charge ordering transition there is a sudden enhancement of $\left\langle S_{1 z} S_{3 z}\right\rangle$. Part of it is attributed to the charge transfer which increases the magnitude of spin moments at the charge rich sites. An AF interaction is expected in the strong coupling limit, $U / t, V / t \rightarrow \infty$, which lead to an effective $J=4 t^{4} / 9 V^{3}$ due to fourth order 'ring' exchange processes 16, 21]. The possibility of having long range order antiferromagnetism (LRO-AF) induced by this AF interaction is explored by allowing AF solutions within CDMFT. As shown in Fig. 6, the staggered magnetization: $m=1 / N_{c} \sum_{i}(-1)^{i}\left(n_{i \uparrow}-n_{i \downarrow}\right)$ becomes finite at the $\mathrm{CO}$ transition, therefore LRO-AF develops concomitanly with $\mathrm{CO}$. This does not discard the possibility of having a paramagnetic COM at finite $T$ as shown previously 22]. This is because the $T$-scale associated with charge ordering $T_{\mathrm{CO}}$ is governed by $V$ while 

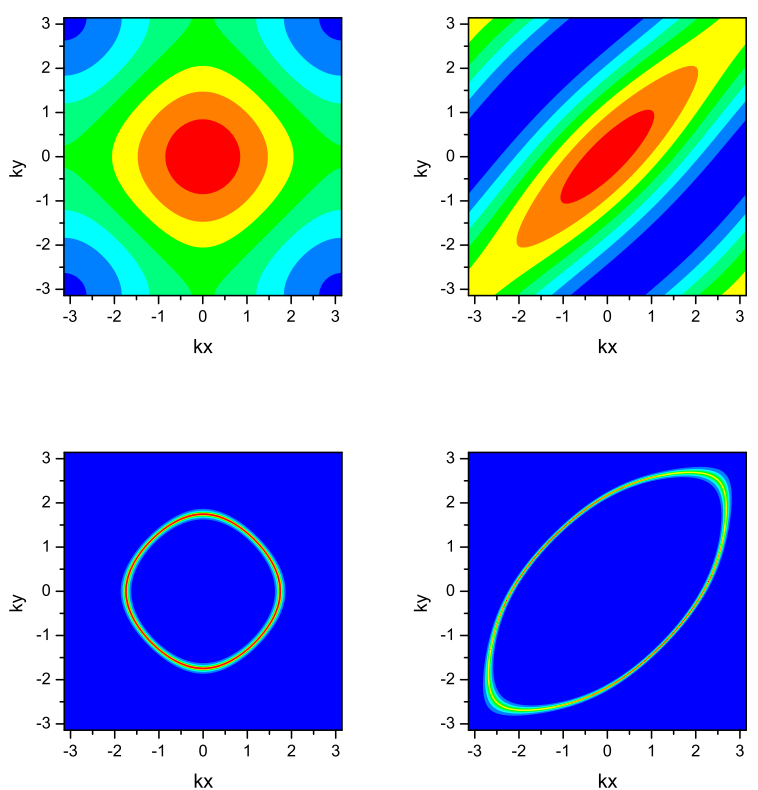

FIG. 5: (Color online) Momentum dependence of spectra close to the MI transition driven by $V$. Top (bottom) figures show the evolution of $1 / \tau_{\mathbf{k}}\left(A\left(\mathbf{k}, 0^{+}\right)\right)$with $V$. Left plots show results for $V=t$ while right plots are for $V=1.8 t$ $(U=10 t)$. Regions with large $1 / \tau_{\mathbf{k}}$ and suppressed $A\left(\mathbf{k}, 0^{+}\right)$ are found close to the COI $(V=1.8 t)$. Blue (red) color correspond to (zero) largest intensities being for bottom left plot: 0 (blue), 1.8 (yellow) and 2.6 (red) and for bottom right: 0 (blue), 1.5 (yellow) and 2.1 (red).
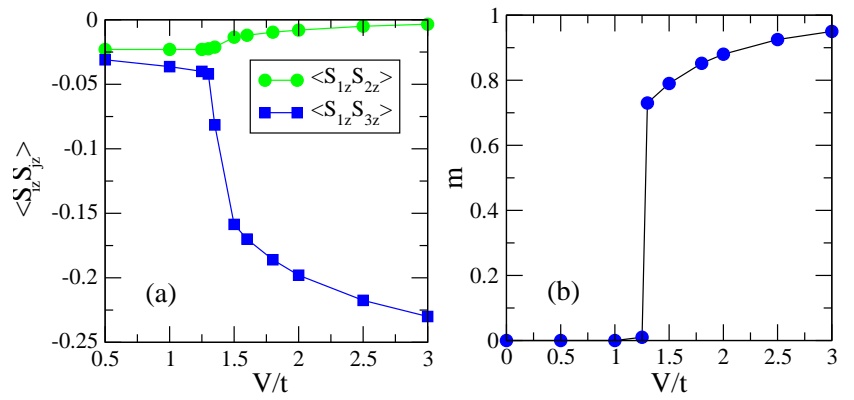

FIG. 6: (Color online) Antiferromagnetic interaction for $U=10 t$. In (a) a sharp increase of the spin-spin correlation, $\left\langle S_{i z} S_{j z}\right\rangle$ between charge rich sites occurs at $V_{C O} \approx 1.3 t$. In (b) the staggered magnetization shows that the onset of LRO-AF coincides with CO.

AF ordering is controlled by $T_{\mathrm{AF}} \sim J<<V$. Hence, the CO paramagnetic metal with a momentum dependent $1 / \tau_{k}$ should exist in the $T$-range: $T_{\mathrm{AF}}<T<T_{\mathrm{CO}}$.

In summary, as the COI transition driven by $V$ is approached the system evolves from a weakly renormalized Fermi liquid to a metal in which the scattering rate becomes momentum dependent which suppresses spec- tral weight on some parts of the Fermi surface. Short range $\mathrm{AF}$ is dynamically generated by $V$ at the $\mathrm{CO}$ transition. Angular resolved photoemission experiments on $1 / 4$-filled $\theta$ and $\beta^{\prime \prime}$ layered organic molecular compounds are highly desirable to test CDMFT predictions.

J. M. acknowledges financial support from the Ramón y Cajal program from MCyT in Spain and MEC under contract CTQ2005-09385. I thank M. Ogata for allowing computer time at University of Tokyo, and M. Civelli, S. Kancharla, E. Koch, G. Kotliar, A. Liebsch, R. H. McKenzie and H. Seo for helpful discussions.

[1] N. F. Mott, Metal Insulator Transitions (Taylor and Francis, London, 1990).

[2] T. Ishiguro, K. Yamaji, and G. Saito, Organic Superconductors (Springer, 2nd Edition, 2001).

[3] F. Kagawa, K. Miyagawa, and K. Kanoda, Nature 436, 534 (2005).

[4] M. Imada, A. Fujimori, and Y. Tokura, Rev. Mod. Phys. 70, 1039 (1998).

[5] A. Georges, G. Kotliar, W. Krauth, and M. J. Rozenberg, Rev. Mod. Phys. 68, 13 (1996).

[6] G. Kotliar, et. al., Rev. Mod. Phys. 78, 865 (2006).

[7] J. E. Eldridge et. al. Sol. Stat. Commun. 79, 583 (1991); D. Faltermeier, et. al., cond-mat/0608090.

[8] B. Kyung, et. al., Phys. Rev. B 73, 165114 (2006); B. Kyung and A. M. S. Tremblay, Phys. Rev. Lett. 97, 046402 (2006).

[9] Th. Maier, M. Jarrell, T. Pruschke, and M. Hettler, Rev. Mod. Phys. 77, 1027 (2005).

[10] H. Seo, J. Merino, H. Yoshioka, and M. Ogata, Jour. Phys. Soc. Jpn. 75, 051009 (2006); H. Seo, J. Phys. Soc. Jpn. 69 805, (2000).

[11] J. Merino, A. Greco, N. Drichko, and M. Dressel, Phys. Rev. Lett. 96, 216402 (2006); N. Drichko, et. al., Phys. Rev. B 74, 235121 (2006).

[12] J. Dong, et. al. Phys. Rev. B 60, 4342 (1999).

[13] S. S. Kancharla, et. al., cond-mat/0508205.

[14] C. J. Bolech, S. S. Kancharla, and G. Kotliar Phys. Rev. B 67, 075110 (2003)

[15] A. I. Lichtenstein and M. I. Katsnelson, Phys. Rev. B 62, R9283 (2000); O. Parcollet, G. Biroli, and G. Kotliar, Phys. Rev. Lett. 92, 226402 (2004); M. Civelli, M. Capone, S. S. Kancharla, O. Parcollet, and G. Kotliar Phys. Rev. Lett. 95, 106402 (2005).

[16] R. H. McKenzie, J. Merino, J. B. Marston, and O. P. Sushkov, Phys. Rev. B 64085109 (2001).

[17] A. Liebsch and T. Costi, Eur. Phys. Jour. B 51, 523 (2006).

[18] A. Yamasaki, M. Feldbacher, Y. F. Yang, O. K. Andersen, and K. Held Phys. Rev. Lett. 96, 166401 (2006).

[19] B. Valenzuela and M. A. H. Vozmediano, Phys. Rev. B 63, 153103 (2001).

[20] R. Pietig, R. Bulla, and S. Blawid, Phys. Rev. Lett. 82, 4046 (1999).

[21] Y. Ohta, K. Tsutsui, W. Koshibae, and S. Maekawa, Phys. Rev. B 50, 13594 (1994).

[22] K. Hanasaki and M. Imada, Jour. of Phys. Soc. Jpn. 74, 2769 (2005). 